\title{
Estudo Comparativo das Características Clínicas e Abordagem de Pacientes com Fibromialgia Atendidos em Serviço Público de Reumatologia e em Consultório Particular
}

\author{
Comparative Study of the Clinical Features and Approach of the \\ Patients with Fibromyalgia Assisted at the Rheumatology Outpatient \\ Clinic and at Physician Private Practice
}

\author{
José Eduardo Martinez ${ }^{(1)}$, Claudia Panossian ${ }^{(2)}$ Fernanda Gavioli(2) $^{(2)}$
}

\section{RESUMO}

Introdução: a fibromialgia (FM) é uma síndrome dolorosa crônica associada a pontos dolorosos à dígito-pressão muscular. São sintomas acompanhantes: fadiga, distúrbio do sono, cefaléia crônica, síndrome do intestino irritável, entre outras manifestações. Ocorre uma influência emocional importante relacionada à resposta anormal ao estresse. A escolaridade, nível socioeconômico relacionado à renda familiar e mesmo a forma de acesso ao serviço de saúde poderiam influenciar a apresentação clínica. Objetivo: determinar se há diferenças significantes na apresentação clínica, intensidade de sintomas e tipo de tratamento entre pacientes atendidos em serviço público e privado de saúde e determinar se há correlação entre a intensidade dos principais sintomas, a renda familiar e a escolaridade das pacientes estudadas. Pacientes e Métodos: Estudou-se 80 pacientes que preenchem os critérios de classificação para FM do American College of Rheumatology (ACR) divididos em dois grupos, de acordo com o local de atendimento: 40 pacientes no ambulatório de reumatologia do Conjunto Hospitalar de Sorocaba e 40 pacientes no consultório privado de um dos autores. As variáveis clínicas foram medidas com escalas analógicas numéricas $(0-10)$ para dor, fadiga, ansiedade, depressão e qualidade de vida. A renda familiar foi estabelecida pelo número de salários mínimos aferidos mensalmente e a escolaridade pelo número de anos de educação formal completados. A comparação entre os grupos foi analisada através dos testes do Qui-Quadrado, ANOVA e diferença de médias. Resultados: os grupos estudados foram semelhantes em relação à intensidade da dor, fadiga, ansiedade, depressão, número de pontos dolorosos, prática de exercícios físicos regulares e duração da doença. Diferiram, porém, em relação à idade (mais jovens no serviço privado) e quanto à renda familiar e escolaridade (maiores no serviço privado). A correlação entre a intensidade dos sintomas

\section{ABSTRACT}

Introduction: Fibromyalgia is a painful chronic syndrome associated to tender areas on digital palpation. Its origin is unknown and probably related to a dysfunction on pain processing pathways. The most frequent accompanying symptoms are: fatigue, sleeping disorders, chronic migraine, irritable bowels syndrome and others disorders. There is an important emotional influence related to stress response. Education, social and economical status could influence clinical presentation. The main purpose of the present study are to determine if there are differences in clinical presentation, symptoms intensity and type of treatment among patients treated in public or private health systems and establish if there is a correlation among main symptoms intensity, family income and formal education status. Patients and Methods: Eighty patients that fulfilled the American College of Rheumatology (ACR) Classification Criteria for Fibromyalgia had been studied. They were divided in two groups according to treatment location: 40 patients have been assisted at the Rheumatology Outpatient Clinic of Sorocaba Hospital Complex (SHC) and 40 patients at one of the authors' private practice. Clinical variables (pain, fatigue, anxiety, depression and quality of life) were measured through a numerical analogic scales $(0-10)$. Family income was evaluated according to the number of minimum wages earned monthly and education according to complete years of formal education. Comparison among groups was analyzed using Chi-square, ANOVA tests and difference among average values. Results: Studied groups were similar regarding to pain, fatigue, anxiety and depression intensity, and number of painful spots, regular practice of physical exercises and disease duration. There have been differences related to age

Pontifícia Universidade Católica de São Paulo (PUC-SP), Conjunto Hospitalar de Sorocaba. Recebido em 3/11/2005. Aprovado, após revisão, em 10/02/06.

1. Professor Titular do Departamento de Medicina da PUC-SP.

2. Acadêmicas de Medicina.

Endereço para Correspondência: Rua Riachuelo, 460/103, Sorocaba, CEP 18035-330, SP, Brasil, telefone: (15) 3224-5603, e-mail: jemartinez@sorocaba.pucsp.br 
e renda familiar ou escolaridade não se mostrou diferente do ponto de vista estatístico. Conclusão: não houve relação entre a apresentação clínica da FM e nível socioeconômico determinado pela renda familiar nem a escolaridade formal. Observou-se associação entre o acesso ao serviço assistencial privado e menor idade de instalação da doença. Isso pode ter ocorrido por facilidade de assistência médica mais precoce nesse grupo.

Palavras-chave: fibromialgia, renda familiar, escolaridade, qualidade de vida.

\section{INTRODUÇÃO}

A fibromialgia (FM) é uma síndrome dolorosa de etiopatogenia desconhecida que acomete preferencialmente mulheres, caracterizada por dores musculares difusas, sítios dolorosos específicos associados, freqüentemente, a distúrbios do sono, fadiga, cefaléia crônica e distúrbios psíquicos e intestinais funcionais. Esta síndrome pode se apresentar isoladamente ou associada a outras síndromes ou doenças clínicas ou mesmo reumatológicas, como hipotireoidismo, lúpus eritematoso sistêmico, artrite reumatóide e outras. ${ }^{(1)}$

Os sintomas incluem dores musculoesqueléticas difusas, distúrbios do sono, fadiga, rigidez matinal de curta duração, sensação de edema, e parestesias. A associação com outras síndromes de natureza funcional é uma constante. Entre elas podemos citar depressão, ansiedade, cefaléia crônica e a síndrome do cólon irritável. Essa sintomatologia se altera em intensidade de acordo com algumas condições, ditas fatores moduladores. Entre elas, a literatura cita mais freqüentemente alterações climáticas, grau de atividade física, estresse emocional, entre outras ${ }^{(2,3,4)}$.

Quanto ao exame físico, a única alteração característica é a hipersensibilidade dolorosa à dígito-pressão em áreas musculares circunscritas e específicas. O número de pontos a ser pesquisado varia, de acordo com alguns autores, entre 12 e 24 áreas ${ }^{(2,3,4)}$. O comitê multicêntrico para a classificação da FM do American College of Rheumatology (ACR) padronizou a pesquisa de 18 áreas musculoesqueléticas circunscritas $^{(5)}$.

Um aspecto importante é o impacto na qualidade de vida dos pacientes e familiares. Os principais determinantes deste impacto são os sintomas de dor e fadiga e a incapacidade funcional resultante ${ }^{(5)}$.

A abordagem destes pacientes deve levar em conta os diversos aspectos destas síndromes, ou seja, o envolvimento muscular, psicocomportamental e social. Entendemos que um protocolo de atendimento multidisciplinar longitudinal (younger people in private system) and regarding to familiar income and education (higher in private system). Correlation among symptoms intensity and family income or education showed no statistical differences. Conclusion: There has been no observed relation among fibromyalgia clinical presentation and social or economical status. It was observed association between private assistance service access and lower age of disease installation.

Keywords: fibromyalgia, family income, quality of life.

é o mais adequado.

Embora a literatura não registre relação entre a sintomatologia fibromiálgica e a renda familiar, diferenças socioculturais poderiam influenciar uma síndrome cujas características têm alto grau de subjetividade. Ainda o grau de dificuldade ao acesso a serviços de saúde pode selecionar casos mais graves em unidades especializadas de saúde, em especial no serviço público.

Em princípio, a população atendida em serviço público tem diferenças em relação à atendida em serviço privado. Diferença em renda familiar, acesso espontâneo ou exclusivamente referenciado são algumas das características que poderiam distinguir essas populações.

\section{OBJETIVOS}

1 - Determinar se há diferenças significantes na apresentação clínica, intensidade de sintomas e tipo de tratamento entre essas duas populações do serviço público e privado. 2 - Determinar se há correlação entre as variáveis clínicas e a renda familiar e o nível educacional na população estudada.

\section{PACIENTES E MÉTODOS}

Foram avaliadas 40 pacientes do sexo feminino, acompanhadas no ambulatório de FM da Disciplina de Reumatologia da Pontifícia Universidade Católica de São Paulo (PUC-SP), atendidas no Conjunto Hospitalar de Sorocaba (CHS) e 40 pacientes acompanhadas no consultório privado do autor principal, tanto particulares quanto conveniados. Todas as pacientes preencheram os critérios de classificação para $\mathrm{FM}$ do $\mathrm{ACR}^{(6)}$. As pacientes foram divididas no grupo A, quando provenientes do CHS, e no grupo $\mathrm{B}$, quando oriundas do consultório privado.

Os dados foram coletados através de entrevistas realizadas nos dias de consultas de rotina e nos prontuários das respectivas unidades. Os seguintes parâmetros de de- 
sempenho foram avaliados:

Avaliação clínica realizada pelo médico para verificar o preenchimento dos critérios de classificação do ACR e o número de pontos dolorosos ${ }^{(6)}$.

Dor, fadiga, ansiedade e depressão foram avaliadas através de escala analógica numérica, de 0 a $10(0=$ ausência do sintoma e $10=$ pior sintoma possível $)^{(5,7,8)}$. A qualidade de vida foi também quantificada através de escala analógica numérica de 0 a 10 ( 0 = maior insatisfação possível com a qualidade de vida e $10=$ maior satisfação possível com a qualidade de vida).

A renda familiar foi avaliada através do número de salários mínimos vigentes auferidos pelos membros da família e a escolaridade avaliada pelo número de anos de educação formal completados. Consideraram-se educação formal os ensinos fundamental, médio e superior.

Obtiveram-se ainda dados demográficos, através de questionário idealizado pelos autores especialmente para essa pesquisa. A análise estatística teve sua metodologia estabelecida pelo Serviço de Bioestatística da Faculdade de Ciências Médicas de Sorocaba da PUC-SP.

Esse protocolo, bem como o consentimento pós-informado foi aprovado pelo Comitê de Ética e Pesquisa do Centro de Ciências Médicas e Biológicas da PUC-SP.

\section{RESULTADOS}

A Tabela 1 apresenta comparações demográficas, renda familiar, escolaridade, intensidade dos sintomas, número de pontos dolorosos e percepção da qualidade de vida entre os pacientes atendidos no serviço público e no serviço privado de reumatologia. Observou-se significância estatística nas variáveis: idade, renda familiar, escolaridade e duração dos sintomas. Não houve diferenças nas variáveis clínicas bem como na satisfação com a qualidade de vida.

Os resultados da análise da correlação entre as variáveis clínicas e a renda familiar não mostraram diferença estatisticamente significante em nenhuma das variáveis $(p>0,05)$. Os resultados da análise da correlação entre as variáveis clínicas e a escolaridade também não mostraram diferença significante em nenhuma das variáveis $(\mathrm{p}>0,05)$.

\section{DISCUSSÃO}

A FM é uma síndrome de natureza crônica e fisiopatologia complexa, que tem na dor musculoesquelética o seu sintoma principal. Sua relação com ansiedade, depressão e exposição aumentada à estressores torna provável que fatores psicossociais influenciem na sua apresentação, evolução,
TABELA 1

DADOS DEMOGRÁFICOS E VARIÁVEIS CLÍNICAS DAS PACIENTES ESTUDADAS

\begin{tabular}{lccc}
\hline VARIÁVEIS & PÚBLIC0 & PRIVADO & $\mathbf{p}$ \\
\hline Idade & $44,3+/-8,37$ anos & $40,1+/-6,80$ anos & $0,01^{*}$ \\
\hline Escolaridade & $8,8+/-0,3$ anos & $9,1+/-0,2$ anos & $0,00^{*}$ \\
\hline Renda familiar & $2,66+/-1,59 \mathrm{sm}$ & $12,15+/-16,90 \mathrm{sm}$ & $0,00^{*}$ \\
\hline Duração & $62,0+/-51,5 \mathrm{~m}$ & $41,3+/-39,3 \mathrm{~m}$ & $0,04^{*}$ \\
\hline Dor* & $8,2+/-1,3$ & $8,3+/-1,7$ & 0,83 \\
\hline Fadiga* & $8,1+/-1,6$ & $7,7+/-1,8$ & 0,31 \\
\hline Depressão* & $6,7+/-3,4$ & $7,6+/-2,3$ & 0,16 \\
\hline $\begin{array}{l}\text { Ansiedade* } \\
\text { No de pontos } \\
\text { dolorosos }\end{array}$ & $7,3+/-2,9$ & $8,1+/-2,0$ & 0,43 \\
\hline $\begin{array}{l}\text { Qualidade de } \\
\text { vida* }\end{array}$ & $14,7+/-2,9$ & $14,5+/-2,73$ & 0,74 \\
\hline
\end{tabular}

adesão ao tratamento, bem como no impacto na qualidade de vida ${ }^{(9)}$.

A proposta desse estudo foi determinar se fatores sociais, educacionais e a forma do acesso aos serviços de saúde poderiam influenciar o quadro clínico e a evolução dos sintomas da FM. Escolhemos duas populações potencialmente diferentes em relação a essas variáveis. Todas as pacientes estudadas estavam em tratamento com medicamentos e orientações estáveis há mais de seis meses. As pacientes vinham sendo atendidas pelo mesmo reumatologista nos dois serviços de saúde, profissional este com experiência no atendimento nessa síndrome.

As pacientes atendidas no CHS foram referenciadas por clínicos de unidades básicas de saúde ou por especialistas de várias áreas de serviços secundários de saúde, tinham seu atendimento custeado pela Secretária de Saúde do Estado de São Paulo e provinham da cidade de Sorocaba e de uma região composta por 48 municípios. A periodicidade de consultas é geralmente quadrimestral e o acesso às unidades de reabilitação é rara, já que a demanda é grande na região em relação à oferta. A atividade física recomendada é realizada através de caminhadas ou em atividade em centros esportivos públicos, geralmente sem a presença de profissional para acompanhar. A medicação é fornecida pelo serviço público, portanto com poucas opções, ou adquirida pelos próprios pacientes quando possível.

As pacientes foram atendidas no consultório do autor principal através de procura espontânea ou com encaminhamento por médicos de outras especialidades ou ainda 
por recomendação de outros pacientes. $\mathrm{O}$ atendimento foi custeado pelo próprio paciente ou através de convênio médico, procedentes da cidade de Sorocaba e de cidades vizinhas. A periodicidade de consultas é variável de acordo com a avaliação do médico ou desejo dos pacientes entre um e três meses e o acesso à unidades de reabilitação e mesmo para outros profissionais de saúde, tais como psicólogos e acupunturista, é geralmente facilitada. A atividade física é realizada através de caminhada, academias de ginástica ou clubes particulares, onde freqüentemente se encontra profissionais de educação física para orientação. As pacientes têm acesso à maior variedade de medicamentos.

Essas diferenças poderiam afetar a apresentação clínica inicial ou pelo menos a evolução ao longo do tempo. Após seis meses de acompanhamento, com tratamento estável, poderia se supor que os grupos evidenciassem essa influência. Isso não ocorreu, e podemos questionar, entre outras possibilidades, que ou a diferença de orientação, atendimento médico, variedade de medicação, estrutura para atividade física não garantem maior ou menor eficiência no tratamento, ou que, mesmo com essas facilidades, as pacientes não as utilizam plenamente.

Nos serviços onde esse estudo se realizou é dada bastante ênfase nas abordagens não-medicamentosas e mesmo na multiprofissionalidade, além de haver uma orientação extensa aos pacientes sobre a síndrome. $\mathrm{O}$ que se pode observar é que apesar das maiores facilidades para adesão a essas medidas no serviço privado, o estado clínico nas duas amostras estudadas não se mostrou diferente, sendo semelhante independentemente da estrutura, poder aquisitivo e nível intelectual das pacientes.

O nível de renda familiar, como indicador de nível socioeconômico, poderia influenciar a apresentação da fibromialgia em relação à intensidade dos sintomas, já que poderia refletir maior disponibilidade de acesso à assistência médica. Esse acesso resultaria, portanto, em diagnóstico mais precoce, consultas com especialistas e outros profissionais da saúde, ou ainda maior facilidade à aquisição de medicamentos e terapias não-medicamentosas.

Outra questão a ser discutida é a relação entre possivelmente maior número de estressores enfrentados por populações de baixa renda comparada àquela de maior poder aquisitivo. $\mathrm{O}$ indicador central de qualidade de vida

\section{REFERÊNCIAS}

1. Goldenberg DL: Fibromyalgia syndrome: an emerging but controversial condition. JAMA 257: 2782-7,1987. é a medida de quanto se está satisfeito com sua própria vida. Esse indicador inclui a saúde, o ambiente, recursos econômicos, relações sociais, bem como atividades relacionadas ao trabalho e lazer ${ }^{(10)}$. Vários estudos mostram que fatores psicossociais, em especial situações repetidas de estresse psicológico, influenciam no desenvolvimento da FM e mesmo no comportamento de procura à assistência médica. ${ }^{(11)}$

A igual freqüência de FM entre populações de rendas diferentes já havia sido observada. A maioria dos estudos sobre FM se originou de países com bons sistemas de saúde ou benefícios sociais adequados. Por outro lado, provavelmente a FM ocorre em igual proporção em população menos favorecida do ponto de vista socioeconômico. A melhor condição social oferece maior variedade de opções de tratamento, em especial naquelas modalidades não-medicamentosas. A falta dessas condições leva a tentativa de alívio dos sintomas em terapias não-tradicionais e, muitas vezes, sem base científica. ${ }^{(11)}$

A escolaridade também poderia alterar a apresentação clínica porque, teoricamente, daria aos pacientes melhores condições para entender o processo da doença, bem como a importância de ajustes no estilo de vida que auxiliariam no enfrentamento da FM, reduzindo a ansiedade que se associa a um pior prognóstico dessa síndrome. Estilo de vida reflete hábitos de atividade física, regularidade de refeições, tabagismo e uso de café e álcool. A literatura apresenta resultados conflitantes em relação à associação entre FM e nível educacional, havendo trabalhos que a associam a menor escolaridade enquanto outros à maior escolaridade. ${ }^{(12,13)}$

A influência do estilo de vida no desenvolvimento de ansiedade e depressão é comprovada. Esses distúrbios podem determinar subgrupos de pacientes com fibromialgia com maior gravidade e maior impacto na qualidade de vida $^{(10)}$. Sabe-se que o uso de estratégias de enfrentamento da FM é geralmente terapêutico e pode reduzir a intensidade dos sintomas. Entre essas estratégias, inclui-se exercícios, meditação, oração, conversas em grupos, e outras atividades. $^{(14)}$

Conclui-se que não há associação entre a apresentação clínica da FM de pacientes em tratamento e renda familiar ou nível de escolaridade.

2. Yunus MB, Masi AT, Calabro JJ, Miller KA, Feigenbaum, SL: Primary fibromyalgia (fibrositis): clinical study of 50 patients with matched normal controls. Semin Arthritis Rheum 11: 151-71, 1981. 
3. Clarck S, Campbell SM, Forehand ME, Tindall EA, Bennet RM: Clinical characteristics of fibrositis. Arthritis Rheum 28: 132-7, 1985.

4. Martinez JE, Atra E, Ferraz MB, Silva PSB: Fibromialgia: aspectos clínicos e socioeconômicos. Rev Bras Reumatol 32: 225-30,1992.

5. Martinez JE, Ferraz MB, Sato EI, Atra E: Fibromyalgia vs Rheumatoid arthritis: a longitudinal comparison of quality of life. J Rheumatol 22: 201-4, 1995.

6. Multicenter Criteria Commitee: The American College of Rheumatology 1990 Criteria for Classification of Fibromyalgia. Arthritis Rheum 33: 169-72,1990.

7. Ledingham J, Doherty S, Doherty M: Primary fibromyalgia syndrome-an outcome study. Br J Rheumatol 33: 139-42, 1993.

8. Wolfe F, Anderson J, Harkness D et al: Health status and disease severity in fibromyalgia. Arthritis Rheum 40: 1571-9, 1997.
9. Martinez JE, Ferraz MB, Fontana AM, Atra E: Psychological aspects of Brazilian women with fibromyalgia. J Psychossomatic Res 39: 197-74, 1995.

10. Kurtze N, Gundersen KT, Svebak S: Quality of life, functional disability and lifestyle among subgroups of fibromyalgia patients: the significance of anxiety and depression. Br J Medical Psychology 72: 471-84, 1999.

11. Alarcon GS, Bradley LA: Coming out of the closet: fibromyalgia in the 1990's. An American Perspective. Rev Bras Reumatol 34: 49-52, 1994.

12. Mäkela $M$, Heliövaara $M$ : Prevalence of primary fibromyalgia in the Finnish population. BMJ 303: 216-9, 1991.

13. Cathey MA, Wolfe F, Kleinheksel SM, Hawley DJ. Socioeconomic impact of fibrositis: a study of 81 patients with primary fibrositis. Am J Med 81 (suppl 3A): 78-84, 1986.

14. Bernard AL, Prince A, Edsall P: Quality of life issues for fibromyalgia patients. Arthritis Care Res 13:42-5, 2000. 